Originalveröffentlichung in: Gockel, Bettina (Hrsg.): Vom Objekt zum Bild : piktorale Prozesse in Kunst und Wissenschaft, 1600 - 2000, Berlin 2011, S. 92-121 (Zurich studies in the history of art ; special issue)


Abb. 1: Rembrandt Harmensz. van Rijn, Die Muschel, 1. Zustand, 1650, Radierung und Kaltnadel, $9.7 \times 12,9 \mathrm{~cm}$, Amsterdam, Rijksmuseum, Rijksprentenkabinet.

Abb. 2: Rembrandt Harmensz. van Rijn, Die Muschel, 2. Zustand, 1650, Radierung und Kaltnadel, $9,7 \times 13,1 \mathrm{~cm}$, Amsterdam, Rijksmuseum, Rijksprentenkabinet. 


\section{Rembrandts Muschel - Nachahmung der Natur? Ein methodisches Lehrstück}

Werner Busch

\section{Der Gegenstand}

Die traditionellerweise Muschel genannte Grafik ist signiert und datiert: „Rembrandt f. 1650 «. Es existieren drei Zustände. Die Technik besteht in Radie. rung, Kaltnadel und Grabstichel, die Masse sind $97 \times 131 \mathrm{~mm}$. Vom ersten Zustand finden sich fünf Exemplare, vom zweiten elf in öffentlichen Sammlungen (Abb.1 und 2), der dritte existiert nur in einem Abzug in Amsterdam mit minimalen Änderungen, er ist im Folgenden zu vernachlässigen. [1] Das Gehäuse der dargestellten Schnecke wirft einen starken Schatten nach links, das niedrige Gewinde verläuft falsch herum, nicht wie bei allen Schnecken nach dem Uhrzeigersinn. Die Siphonalrinne zur Basis verläuft also spiegelverkehrt. Es handelt sich um Conus marmoreus aus dem Indopazifik, zur Familie der Conidae gehörig. Ihre Beschreibung lautet: Zwischen fünf und zehn Zentimeter Gehäusehöhe, Schulterkante schwachknotig, flache oder leicht konkave Rampe (sogenanntes Analband), hell-dunkle Gitterzeichnung, dunkles Braun mit weissen, zumeist dreieckigen Flecken. ${ }^{[2]}$ Schatten und falsche Richtung des Gewindes lassen den Schluss zu, dass Rembrandt, wie gelegentlich, die Umkehrung durch den Druckvorgang für die Darstellung des Gegenständlichen nicht bedacht oder nicht für wichtig erachtet hat - im Gegensatz zu Signatur und Datierung, die im Druck richtig erscheinen. ${ }^{|3|}$

Der erste Zustand zeigt allein den Schneckenkörper und den Schatten. Er fällt besonders im Gewindebereich sehr dunkel aus. Die obere Abschlusslinie des Schneckenkörpers wirkt unvollendet, sie lässt Lücken im Umriss. Die Forschung hat dies mehrfach bemerkt und hält die Radierung von daher in diesem Zustand für unvollendet. Wozu allerdings zu sagen ist - Newton hat es später bewiesen -, dass bei einer starken Hell-Dunkel-Abfolge optisch das Weiss wie aus dem Gegenstand herausspringend erscheint. Rembrandt dürfte also eher eine optische Erfahrung wiedergegeben haben.

Der zweite Zustand bringt Aufhellungen im Gewindebereich und an den Aussenlippen des Mündungsrandes. Es findet eine stärkere räumliche Einbindung statt, die allerdings letztlich unklar bleibt. Die Schnecke scheint fachartig gerahmt, die gesamte Fläche innerhalb des Plattenrandes mit Ausnahme eines anderthalb Zentimeter breiten Streifens am unteren Rand, in dem sich auch Signatur und Datierung befinden, ist durch Schraffuren bis zu tiefster Verschattung rechts hinter dem Schneckenkörper strukturiert. Wie wiederum oft bei Rembrandt dient die Nachbearbeitung der Austarierung: Die im ersten Zustand empfundene Verlagerung nach links wird durch den dunklen Schatten rechts aufgehoben. Die Schnecke leuchtet jetzt eher aus dem Dunkel heraus. Die sonderbaren Längsstriche am linken Rand, die man als Holzrahmen lesen könnte, bewirken eine Lektüre von links nach rechts in den Raum hinein. Sie verorten den zuvor ortlosen Gegenstand. Der Fächercharakter entsteht durch die leichte Betonung einer Grundlinie, die in etwa auf Höhe der Siphonalrinne verläuft, was der Darstellung weitere Festigkeit verleiht. Der dritte Zustand, offenbar nach 
schneller Abnutzung der Platte entstanden, bringt geringfügige Aufhellungen und Formpräzisierungen und besonders am Gewinde eine leichte Zuspitzung.

\section{Funktion und Kontext der Grafik}

Es ist nicht einfach zu sagen, welchem Nutz und Frommen die Grafik gedient hat. Die Forschung schlägt Verschiedenes vor: Es soll hier in extenso vorgeführt werden, um das methodische Problem deutlich werden zu lassen. Denn die Fülle der Bedeutungsfacetten konnte offensichtlich nur deswegen an den Gegenstand herangetragen werden, weil er, semiotisch gesprochen, unterdeterminiert ist. So liefern die Ausdeutungen Verständnismöglichkeiten, ohne eine definitive Bedeutung markieren zu können. Es dürfte allein möglich sein, aus dem kulturgeschichtlichen Kontext im Abgleich mit dem Erscheinungsbild der Grafik wahrscheinlichere oder weniger wahrscheinliche Aspekte der Sache namhaft zu machen.

\section{Wenzel Hollar als Anregung}

Als Vorbild wird grundsätzlich eine 38 Blatt umfassende Muschel- bzw. Schneckenserie von Wenzel Hollar, ohne Titel und Signatur, dingfest gemacht, die aus den $1640 e r-J a h r e n$ stammen dürfte. Die Wiedergabe der Gehäuse bei Hollar ist sehr präzise, allerdings werfen sie keinen Schatten (Abb. 3). Dagegen finden sich Licht und Schatten am Gehäusekörper selbst. ${ }^{[4]}$ Ferner sollte man darauf hinweisen, dass sich auf Hollars zwölf Blatt umfassender Serie Mus. carum Scarabeorum zu Faltern, Käfern und Raupen (Abb.4) von 1646 dem Rembrandt'schen Erstzustand entsprechende Schatten finden, ${ }^{[51}$ die im Übrigen in der aquarellierten Tradition zoologischer Darstellungen von Georg Hoefnagel (1542-1600) an durchaus üblich sind und von da auch für gedruckte naturwissenschaftliche Illustrationen übernommen werden, so bei Hoefnagel selbst, etwa in seinem Werk Archetypa studiaque patris, Frankfurt 1592 (Abb.5). ${ }^{[6]}$ Das Argument, wegen des ausgeprägten Schattens könne es sich bei Rembrandt nicht um eine naturwissenschaftliche Illustration handeln, greift also nicht wirklich. Allerdings ist die tenden. ziell atmosphärische Einbindung bei Rembrandt schon im ersten Zustand stärker als in der naturwissenschaftlichen Illustrationstradition üblich. Zudem ist die auch bei Hollar zu konstatierende Isolierung einer Schnecke auf einem Blatt in dieser Tradition zumindest ungewöhnlich. Allerdings findet sich unter Hollars Blättern Conus marmoreus nicht, wenn auch andere Schnecken der Familie der Conacea, der Kegelschnecken, wie Conus imperialis (Abb. 6). ${ }^{\mid 71}$ Sie waren früh begehrt, wurden $» R u h m$ des Meeres", aber auch wegen der hochgiftigen Schnecken, die sie bewohnten, "Giftschlangen des Meeres" genannt. ${ }^{[8]} \mathrm{Zu}$ den besonders giftigen gehört auch Conus marmoreus, ein Stich kann für den Menschen lebensgefährlich sein. In Holland im 17. Jahrhundert wurde der Conus marmoreus, wie ein Aquarell von Bartholomeus Assteyn durch die Beischrift deutlich macht, "Herts Horen«, Hirschhorn, 


\section{Trochins Pyiramidialis.}


Abb. 3: Wenzel Hollar, Kreiselschnecke, 1644/1650, Radierung, $9.4 \times 14.3 \mathrm{~cm}$, Berlin, Kupferstichkabinett der Staatlichen Museen, Preussischer Kulturbesitz.

Abb. 4: Wenzel Hollar, Schnecke und Raupen, 1646, Radierung, 8,0 ×11,7 cm, in: Hollar, Wenzel (1646), Muscarum, Scarabeorum vermiumque variae figurae et formae. Antwerpen: Schenk, Abb. 4, Berlin, Kupferstichkabinett der Staatlichen Museen, Preussischer Kulturbesitz. 



Abb. 5: Jacob Hoefnagel, Sub omni lapide dormitat scorpius, 1592, Kupferstich, $15.3 \times 21,2 \mathrm{~cm}$, in: Hoefnagel, Jacob (1592), Archetypa Studiaque Patris G. Hoefnagelii. Frankfurt: Hoefnagel, Teil II, 7.

Abb. 6: Wenzel Hollar, Conus imperialis, 1644/1650, Radierung, 9,8 $813,6 \mathrm{~cm}$, Amsterdam, Rijksmuseum, Rijksprentenkabinet. 
genannt (Abb.7). ${ }^{[9]}$ Im Übrigen führt dieses Aquarell einen dem Rembrandt'schen Erstzustand nahe verwandten Schatten konsequenterweise nach rechts. Das Blatt gehört zu einer Serie von Illustrationen aus der Sammlung Lugt, zwei Schnecken mit schreibmeisterlich vorgetragener Benennung finden sich übereinander, gelegentlich aber auch Einzeldarstellungen, wie die wundervolle Wiedergabe einer am Faden hängenden "gefleckten platten Krabbe«, wie sie genannt wird und bei der es sich wohl um eine der Vasenschneckenspecies handeln dürfte. ${ }^{\mid 10]}$ Trotz ihres Hängens wirft sie einen schwachen Schatten auf das Papier, das so zur Wand wird (Abb. 8). So ist die Grenze zwischen rein naturwissenschaftlicher IIlustration und bildhafter Präsentation nicht leicht zu ziehen. In den Augen des Sammlers vermischen sich ohnedies das Bedürfnis nach Klassifizierung und dasjenige nach ästhetischem Genuss.

\section{Grafik und Schnecke als Sammelgegenstand}

Rembrandts zweiter Zustand löst die Darstellung endgültig aus der naturwissenschaftlichen Tradition und macht sie zu einem eigenständigen Kunstwerk. Doch was rechtfertigt nun den Gegenstand? Und: Welche Konnotationen verbinden sich inm? Die Forschung bietet vieles in Nuancen an: Die Grafik wird als Grafik zum Sammlerstück. Rembrandt führt oft bewusst rar gemachte verschiedene Zustände einzelner Grafiken auf unterschiedlichen Papieren gedruckt im Angebot, so gut wie jeder Abzug fällt minimal anders aus, da er auch mit Wischungen auf dem
Plattengrund arbeitet. Conus marmoreus selbst war gesuchter Sammelgegenstand, nicht wirklich selten, dafür tauchte er auf zu vielen Gemälden auf, aber doch wertvoll. Schliesslich kam er aus dem Indopa. zifik und fand seinen Platz von daher in Kunstkammern und Kunstkammerbildern (Abb. 9).

\section{Gottes Schöpferreichtum}

Der Gegenstand galt als exotisch, weckte curiositas, war Bestandteil so gut wie aller Kunstkammerdarstellungen. Er vertrat hier die Naturalia im Gegensatz zu den Artificialia und war Exempel des Mikrokosmos, so wie die Kunstkammer selbst einen Mikrokosmos im Rahmen des Makrokosmos verkörpert. Da Schnecken zudem schon früh als Verkörperungen absoluter göttlicher Proportionalität erkannt wurden - Nautilusschnecken etwa galten als nach den Verhältnissen des Goldenen Schnitts, der divina proporzione, gegliedert -, konnten sie in Analogie zum göttlichen Weltenbau gesehen werden. ${ }^{[11]}$ I hre schier unendliche Form- und Farbvarianz, andererseits die Möglichkeit, sie zu Familien zu ordnen, wobei Verwandtschaftsbeziehungen über Tausende von Kilometern hinweg unabweisbar schienen, ferner die verblüffende Präzision hochkomplexer Muster - all dies liess sie geradezu zum Gottesbeweis werden.

\section{Holländischer Reichtum}

Der Besitz von Conus marmoreus war auch Ausdruck von individuellem Reichtum, aber vor allem Ausdruck 



182

Abb. 7: Bartholomeus Assteyn, Herts Horen, Mitte 17. Ih., schwarze Kreide und Aquarell, 31,4 $\times 20,3 \mathrm{~cm}$, Paris, Fondation Custodia, Collection Frits Lugt. 




Abb. 8: Bartholomeus Assteyn, Platte Krabbe, Mitte 17. Jh., Gouache, $31,2 \times 20,2 \mathrm{~cm}$,

Paris, Fondation Custodia, Collection Frits Lugt. 




Abb. 9: Frans Francken II, Kunstkammer, nach 1636, Öl auf Holz, $74 \times 78 \mathrm{~cm}$,

Wien, Kunsthistorisches Museum. 
des Stolzes auf die holländische Beherrschung des Meeres, den holländischen Handelsreichtum, beispielhaft verkörpert in der Ostindischen Kompanie. In Amsterdam wurde ein schwunghafter Handel mit exotischen Muscheln und Schnecken getrieben. Die "Nutzlosigkeit» der Schalen und Gehäuse prädestinierte sie nicht nur als ästhetische Objekte. Sie waren schöner Schein und Zeichen von Überfluss zugleich. Nicht nur darum erscheinen sie auf Kunstkammerbil. dern häufig in Begleitung von antiken Münzen, die nicht mehr Währung bildeten, sondern ebenfalls Sammelgegenstand geworden waren. Die Demonstration von Überfluss ist in einer calvinistischen Gesellschaft nicht unproblematisch. Insofern lässt sich auch hier sagen, die Muscheln und Schnecken können sich nicht in ihrer Funktion, Besitz vorzuführen, erschöpfen. Es gilt, den Reichtum sub specie aeternitatis zu sehen, den Sammelobjekten ein dialektisches Verständnis einzuschreiben, ihren Besitz als irdisches und vergängliches Geschenk zu begreifen.

\section{Naturwissenschaftliche Dimension}

Selbst wenn das Erscheinungsbild des zweiten $\mathrm{Zu}$ standes von Rembrandts Grafik ausschliesst, dass es sich bei seinem Blatt um eine naturwissenschaftliche Illustration handelt, so ist die Darstellung doch naturwissenschaftlich ziemlich genau und konnte im naturwissenschaftlichen Diskurs verschiedenen Argumenten dienen, etwa der theologisch äusserst brisanten Frage nach dem Alter der Erde. Schliess. lich fand man Muscheln und Schnecken auf den
Gipfeln der Berge in versteinerter Form. War dies das Ergebnis der Sintflut und in ihrem Gefolge bewirkter Auffaltungen, konnte es, folgte man den Funden und geologischen Strukturen, nur eine Sintflut, nur eine Auffaltung gegeben haben, oder mussten nicht mehrere angenommen werden, und vor allem in weit vergangenen, im Grunde genommen vorbiblischen Zeiten? ${ }^{\mid{ }^{12)}}$ Selbst wenn die Muscheln und Schnecken erst im 18. Jahrhundert, vor allem durch Carl von Linné, in eine verbindliche Ordnung gebracht wurden. Typenverwandtschaften über weite Strecken hinweg wurden selbstverständlich, wie fehlerbehaf. tet auch immer, schon vorher erkannt. In Michele Mercatis Metallotheca Vaticana ist die gesamte naturkundliche Sammlung des Vatikan dokumentiert, die von Anton Eisenhoit in der zweiten Hälfte des 16. Jahrhunderts gestochen, jedoch erst Anfang des 18. Jahrhunderts publiziert wurde (Abb. 10). Dort findet sich etwa die Gattung der Purpurschnecken, von Plinius bis Linné "Murex" genannt. Sie gehören zu den Stachelschnecken, selbst wenn sie hier auch mit den Walzenschnecken vermischt werden, und produzieren Purpur zum Färben der Gewänder, was ihre Berühmtheit erklärt. Auch Eisenhoit reproduziert sie seitenverkehrt - womit noch einmal ein Argument für Rembrandts naturwissenschaftliche Ungenauig. keit entfällt. ${ }^{[13]}$

\section{Vanitas}

Wie selbstverständlich wurde immerhin noch in den 1980er-Jahren Rembrandts Muscheldarstellung 


\section{Lapides I $\triangle$ IOMOPФOI. \\ L $\quad \mathrm{O} \quad \mathrm{C} \quad \mathrm{U}$ L $\mathrm{O}$ XXXV.}

PORPHIROIDES, ET MURICITES.

\section{A P. XXXXII.}

Pecres Purpurarum, qux demonftrantur, nunquam Curiam Romanam $\checkmark$ depinxerune, non infiguia furgentis ad majeftatcm pueritix prxtule-



PORPHYROIDES

CHoystio, Comiser sculp

runt, nunquam lanas nobilitarune. Qui fecus $x$ ftimat, illuın Natura elufit. Lapides enim funt, atque originem ducunt à fotu terrx. Cuodque admira$P \rho=$ tioncm 
eine definitive Vanitas-Dimension beigemessen. ${ }^{[14]}$ Danach wurde sie ziemlich regelmässig als eine denkbare oder wahrscheinliche Bedeutungsebene begriffen, etwa im Berliner Rembrandt-Katalog von 1991, [15] schon Pennington hatte im Wenzel-HollarWerkverzeichnis 1982 auf die Leblosigkeit und Zerbrechlichkeit der Muscheln hingewiesen und damit auf ihre Vergänglichkeit. ${ }^{[16]}$ Und in der Tat, in Vanitas-Stillleben tauchen nicht selten Muscheln bzw. Schnecken auf. Allerdings sollte man genau hinse. hen, es findet sich eine Art Standardkombination von einem Totenkopf mit einer grossen Schnecke, die zu den Turbinidae, den Kreisel- oder Turbanschnecken, gehört (Abb. 11 und 12). ${ }^{[17]}$ Diese Schnecken können bis zu 20 Zentimeter gross werden. Sie sind dadurch ausgezeichnet, dass sie ein perlmutternes Inneres haben und durch Abschleifen auch äusserlich glänzend perlmuttern erscheinen können, wie auch die Nautilusschnecke, die ja an sich von aussen grossstreifig rötlich-braun verziert ist, doch für die Nautiluspokale fast immer abgeschliffen genutzt wurde, um mehr Glanz zu verbreiten und zur Edelmetallfassung, zumeist Silber, zu passen. Zentral wird sie etwa auf Goltzius' Pictura-Allegorie zur Anschauung gebracht (Abb. 13). ${ }^{[18]}$

Doch die Turbanschnecke ist noch durch eine andere Besonderheit geprägt. Sie hat eine sehr grosse, schlundartige Mündung (Abb.14), die zu bestimmten lahreszeiten mit einem Kalkdeckel verschlossen wird, die schönste Art bildet ihn als buntes, stark blaugrün leuchtendes sogenanntes Katzenauge aus. Dieser Typus, der sich besonders auf den Philippinen findet, wird bis zu acht Zentimeter gross. In den Vanitas. Stillleben tauchen die Turbanschnecken grundsätzlich ohne Deckel auf, und grundsätzlich schauen wir tief in den Schlund, manchmal bis ins Dunkle. In der Kombination mit dem Totenkopf, seinen Augenhöhlen, dem nicht selten fehlenden Unterkiefer, sodass man häufig schräg von unten in die leere Mundhöhle schaut - etwa auf Bildern von Jan Davidsz. de Heem, Adriaen van Nieulandt oder Harmen van Steenwyck -, erscheint auch die Turbanschnecke als vom Leben verlassenes Gehäuse, in dem die Leere und der Tod gähnen. ${ }^{[19]}$ Zum anderen bestehen Muscheln und Schnecken primär aus Kalk. Nicht selten finden sich Exemplare mit deutlichem Abrieb, das Muster droht zu verschwinden. Bei Conus marmoreus ist das häufig an der Schulterkante und der Siphonalrinne der Fall - so kommt unter dem Glanz das blosse Material zum Vorschein und damit die Vergänglichkeit.

\section{Die emblematische Dimension der Muscheln und Schnecken auf Kunstkammerbildern und auf Pictura-Allegorien}

Wir wissen seit Langem, dass die Dinge in der Em. blematik in bono und in malo gedacht werden können. In den Beschreibungen einer zeitgenössischen Kunstkammer wird das Betrachten der Conchylien als vergnüglich und ergötzlich verstanden, sie galten als Zeichen von Gottes wunderbarer Naturschöpfung. ${ }^{[20]}$ In Roemer Visschers Sinnepoppen dagegen, in Amsterdam 1614 zuerst erschienen, wird das modische Muschelsammeln als geradezu 




Abb. 11: Harmen van Steenwijck, Vanitas, 1640, Öl auf Holz, 39, $2 \times 50,7 \mathrm{~cm}$, London, The National Gallery.

Abb. 12: Adriaen van Nieulandt, Vanitas, 1636, Ol auf Leinwand, $39,5 \times 36 \mathrm{~cm}$. Haarlem, Frans Hals Museum.






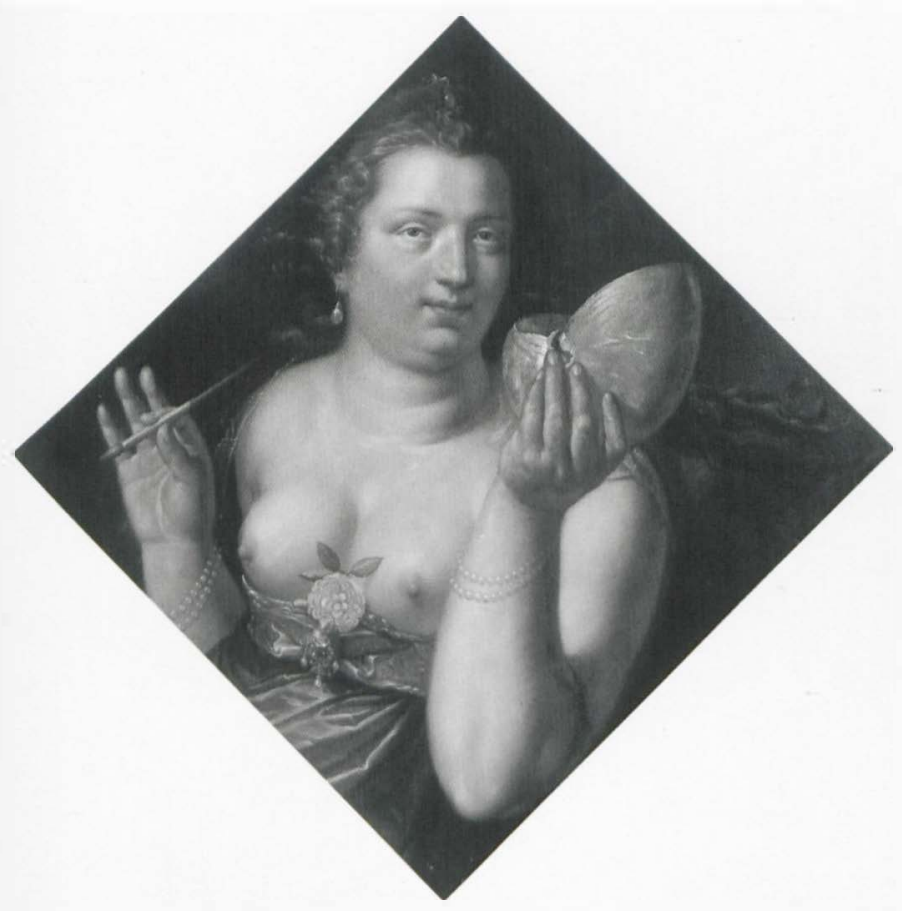

Abb. 13: Hendrik Goltzius, Pictura-Allegorie, 1610, Öl auf Holz, $67 \times 79 \mathrm{~cm}$, Privatsammlung.

Abb. 14: Jan Davidsz. de Heem, Vanitas, Mitte 17. Ih., Öl auf Leinwand, $46 \times 56,5 \mathrm{~cm}$, Privatsammlung.








Abb. 15: Jacques Linard, Stillleben mit Muscheln und Korallen, um 1640 ,

Öl auf Kupfer, $47 \times 64 \mathrm{~cm}$, Paris, Fondation Custodia, Collection Frits Lugt. 
widerwärtig empfunden, nur ein Narr könne dafür sein Geld hergeben. ${ }^{[21]}$ Aber die Schnecken und Muscheln können auch Erkenntnismedium sein, sie können aus dem Kunst- und Wunderkammernzusammenhang, in dem sie Zeugnis von Gottes Grösse, aber auch von des Sammlers göttlicher Ebenbildlichkeit ablegen, herausgelöst und zu reinen Muschelstillleben werden, und dort demonstrieren sie primär die Artenvielfalt, so vor allem bei Balthasar van der Ast und Jacques Linard (Abb.15). Auch Rembrandt hat Muscheln gesammelt, wie sein Insolvenzinventar von 1656 bezeugt: Er besass "a groote quantiteit hoorens [en] seegewassen «, ${ }^{[22]}$ das hat man übersetzt mit "Muscheln und Meerbewohnern«. Man sollte es besser wörtlich übersetzen: Muscheln bzw. Schnecken und Seegewächse. Für Rembrandt waren sie wohl nicht nur "studio-props«, sondern Teil eines eher enzyklopädischen, letztlich patrizischen Anspruchs. Aber auch dieser konnte in den patrizischen Kunst- und Wunderkammern, wie sie Rubens und Jan Brueghel d. Ä. in ihren gemeinsamen Bildern, wie sie Frans Francken II. oder Willem van Haecht in ihren idealen Galeriebildern festgehalten haben, doppelt konnotiert sein, worauf besonders Justus Müller-Hofstede und ihn ergänzend Ekkehard Mai aufmerksam gemacht haben. Müller-Hofstede liest vor allem aufgrund des nicht zu leugnenden gegenreformatorischen Zusammenhangs die Bilder von Frans Francken II. und von Rubens und Brueghel in Bezug auf die Conchylien letztlich in malo. Der Erkenntnisgewinn, den ihre Betrachtung verspricht, ist relativ, kein Selbstwert, erst die Betrachtung sub specie aeternitatis, erst ein Erkenntnisstreben, das auf das Heil gerichtet ist, vermag auch der Naturerkenntnis ihren Ort zu geben. Und so liest er die Bildergalerien - nicht immer ganz schlüssig - von unten nach oben, von niederer zu höherer Erkenntnis, gespiegelt in den Themen der Bilder. Der niederen Sphäre sind dann auch eher die Conchylien zugeordnet. ${ }^{[23]}$ Das mag eine Tendenz der Bilder sein, doch die eher gegenteilige Konnotation lässt sich auch nicht leugnen. Insbesondere die Galerie- bzw. Kunstkammerbilder, die ausdrücklich eine Pictura-Allegorie darstellen, würdigen auch die Conchylien in doppelter Hinsicht; denn nicht immer ist es so, dass die zentralen Bilder im Bilde dem christlichen Überbau dienen. In Frans Franckens Pictura-Allegorie (Abb.16) von 1636 ist das Hauptbild über dem zentralen Buffet den Gelehrten auf dem Parnass gewidmet, ihr Ruhm wird verkündet, die Götter residieren über ihnen. Die Wissenschaften als Freie Künste vertreiben die lasterhafte Ignoranz, sie wird ins Reich der Finsternis zurückgetrieben. Unmittelbar vor dem Gemälde, auf der Anrichte, genau in der Breite des Gemäldes sind Conchylien "angerichtet«, als wären sie besonders geeignetes Erkenntnismedium der Gelehrten im Bilde. ${ }^{[24]}$ Und zum anderen dürfte das alte Winner'sche Argument greifen, der in allen Galeriebildern mehr oder weniger verkappte PicturaAllegorien sieht. ${ }^{\{25\}}$ Gegenreformation hin, Gegenreformation her, hier werden offenbar auch die Malerei und ihre Fähigkeit, alles überzeugend darstellen zu können, gefeiert. Der Malerei als Ars gebührt 
Schöpferrang, da sie in der Lage ist, auch die Naturalia im Bilde zu verewigen - was ihr, die rhetorische Dichotomie oder Dialektik wird man schlicht hinnehmen müssen, in diesem oder jenem Kunstkammerlob gerade bestritten wurde. Selbst die grössten Künstler könnten es, so heisst es dort, nicht mit der Farbenpracht der göttlichen Naturgebilde aufnehmen. ${ }^{[26]}$ Und so ist es wohl auch mit der grossen Fliege auf dem Buch im Vordergrund von Franckens Galeriebild, auf dem sich sein Bildnis, seine Signatur und sein Motto befinden. Weniger die in der Literatur bemühte emblematische Fliege der gänzlichen Ignoranz, wie sie in Sambucus' Emblemata 1566 beschrieben ist, dürfte gemeint sein, ${ }^{[27]}$ sondern schlicht ein Trompe-l'đeil in Plinius'scher Tradition. Man möchte sie wegscheuchen, so "echt « soll sie erscheinen, eben darum ist sie allerdings auch grösser wiedergegeben als der Realität des Bildes angemessen. Sie soll vermeintlich unserer Sphäre angehören und von der besonderen Kunstfertigkeit des Künstlers Zeugnis ablegen. Sie, die Fliege, hat das Bild nicht als Bild erkannt, eine Erkenntnis, zu der wir im Zuge der Betrachtung dagegen gelangen sollen. Das Motiv von Täuschung und Enttäuschung zugleich ist aufgerufen. ${ }^{[28]}$

\section{Die Muschel im Meer und im Paradies}

Auch Rembrandts sogenannte Muschel ist als Nachweis besonderer Kunstkompetenz zu lesen, schon dadurch, dass der Künstler in offensichtliche Kunstkonkurrenz zu Wenzel Hollar tritt: Das bloss
Naturrichtige wird bildhaft nobilitiert. Nun hat Rembrandt noch einmal eine Muschel dargestellt, und zwar an überraschender Stelle: im Vordergrund seines Hundertguldenblattes, von dem gemeinhin angenommen wird, dass es 1649 , also im Jahr vor der Fertigung von Conus marmoreus, vollendet war (Abb. 17). Die Muschel liegt hier scheinbar ohne jeden sinnvollen Zusammenhang im Vordergrund, begleitet allein links von einem abgebrochenen Zweig. Auf den ersten Blick macht das im Zusammenhang von Matthäus 19, dem die Szene verpflichtet ist, keinen Sinn. Rembrandt rekurriert bekanntlich auf das gesamte biblische Kapitel: Das dort sukzessiv Geschilderte wird hier simultan zur Anschauung gebracht. ${ }^{[29]}$ Doch vielleicht ist ein etwas weit hergeholter Gedanke erlaubt. Muschel und Zweig liegen am Rand eines schwer auszumachenden, aber doch beinahe die ganze Bildbreite durchmessenden Erdspaltes, den man vielleicht als ein ausgetrocknetes Bachbett lesen kann oder auch als vertrocknete Quelle. So wie sich der Strom der Hilfesuchenden zu Christus ergiesst, so könnte die vertrocknete Quelle auf die zweifelnden Pharisäer und Schriftgelehrten verweisen. Zu diesem Gedanken kann eine ganz andere Darstellung führen: Hugo van der Goes' Sündenfall (Abb.18) aus dem Wiener Diptychon. ${ }^{[30]}$ Dort nämlich, rechts im Vordergrund neben der Schlange der Versuchung, ist ein schmaler Bach zu sehen, offenbar die Quelle des Paradiesesstromes, und in ihm liegen nahe dem vorderen Bildrand Muschel und Zweig im Wasser, der Zweig ist hier eindeutig eine Koralle. Die Muschel bei Hugo van der Goes im 




Abb. 16: Frans Francken II, Allegorie der Pictura, 1636, Öl auf Holz, $92 \times 123 \mathrm{~cm}$,

Privatsammlung, ehem. Johnny Van Haeften Ltd. London. 




Abb. 17: Rembrandt Harmensz. van Rijn, Hundertguldenblatt, 1. Zustand, um 1648,

Radierung und Kaltnadel, $27,8 \times 38,8 \mathrm{~cm}$, Amsterdam, Rijksmuseum, Rijksprentenkabinet. 




Abb. 18: Hugo van der Goes, Sündenfall (vom Wiener Diptychon), 1477, Öl auf Holz, 32,3×21,9 cm,

Wien, Kunsthistorisches Museum. 
Übrigen ist ein Meerohr, in dieser Grösse als sogenanntes Grünes Meerohr mit einer Kette auch hier sichtbarer Atemlöcher eigentlich nur an der kalifornischen Küste zu finden, in etwas kleinerer Form jedoch auch im Indischen Ozean oder im Pazifik. ${ }^{[31]}$ So wird man sagen müssen, schon im 15. Jahrhundert sind Künstler in der Lage, exotische Funde naturkundlich richtig darzustellen. Ihre ungewöhnliche Erscheinung jedoch soll offensichtlich darauf verweisen, dass sich in ihrem Zitat tieferer Sinn verbirgt. Muschel und Koralle in ihrer Kombination machen in der nachfolgenden ikonografischen Tradition den Inbegriff der Meeresschätze aus. Auf Jacopo Zucchis dem Reichtum des Meeres gewidmetem Bild in der Galleria Borghese hebt im Zentrum die Königin des Meeres, Amphitrite, mit der Rechten eine perlenbesetzte Muschel hoch, mit der Linken ein sechsstrahliges Korallenbäumchen (Abb. 19). ${ }^{[32]}$ In den Kunst- und Wunderkammerbildern, auch in Erdteildarstellungen oder in "Neptun und Amphitrite«-Bildern tauchen Muscheln und Korallen regelmässig zusammen auf. Und um die Bedeutung des Motivs zu betonen, wird anstelle einzelner Perlen häufig eine ganze Perlenkette dazugelegt, die etwa bei Frans Francken II. von Nereiden zusammen mit Muscheln und Korallen dargeboten wird. In Jan van Kessels Amerika- und Afrikadarstellungen (Abb. 20) finden sie sich entsprechend auf dem Boden ausgebreitet. ${ }^{[33]}$ Muschelperlen und Korallen sind emblematisch vielfach, vor allem mariologisch und moralisch, aufgeladen. Die Koralle, so will es die Emblematik in verschiedenen Varianten, ist, direkt aus dem Wasser geholt, zuerst weich, wässrig und unscheinbar, doch an der Luft wird sie hart, gewinnt Farbe und wird schön. In Saavedras Emblembuch steht sie folgerichtig unter dem Motto »Robur et decus «, »Kraft und Schönheit«. ${ }^{[34]}$ Sehrviel weiter hilft diese Ausdeutung in unserem Zusammenhang allerdings nicht. Offenbar ist unter anderem die alte Reflexion über Innen und Aussen gemeint, wobei mal das eine, mal das andere positiv respektive negativ gesehen werden kann. Da der Reichtum der Meere nicht selten auch mit Mineralogischem gekoppelt wird - das eine entstammt den Meerestiefen, das andere der Tiefe der Erde, in barocken Grotten gewinnt ihre Synthese ihre anschaulichste Gestalt -, handelt es sich wohl um die Betonung von Gottes Schöpfung in ihrer elementaren Form. ${ }^{[35]}$

\section{Schaulust}

Es wären noch weitere Dimensionen zu benennen. So ist etwa von der Bedeutung der Form vor allem der Schnecken noch nicht die Rede gewesen. Ihre Spiralform kann als Wachstumssymbol, auch als Weltenordnungsanalogie verstanden werden, die Muschel kann mit Venus, aber auch der Taufe zusammengebracht werden. Piero Valeriano lässt die Schnecke dem Weltlichen verfallen sein, Filippo Buonanni nennt eine erste reine Conchyliologie 1689 Ricreatione dell'occhio e della Mente nell'Osservatione delle Chiocciole und lässt auf dem Titelblatt eine melancholische Frau am Ufer über einer Muschel mit Perle reflektieren, auf dem Meere herrscht Neptun (Abb. 21). ${ }^{[36]}$ Obwohl der Herausgeber Jesuit ist, geht 


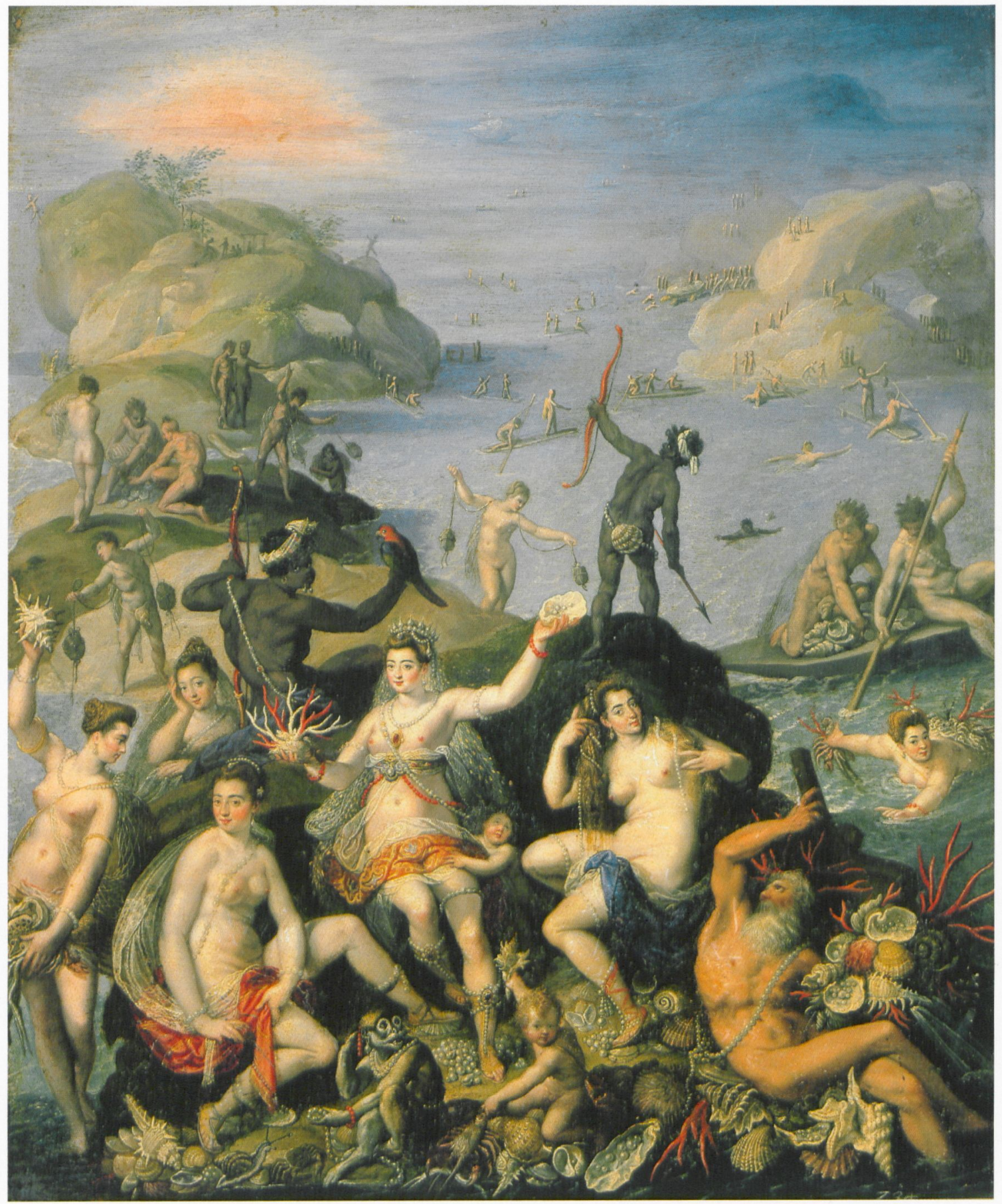

Abb. 19: Jacopo Zucchi, Korallenfischer, 1585 , Öl auf Kupfer, $55 \times 45 \mathrm{~cm}$,

Rom, Galleria Borghese. 


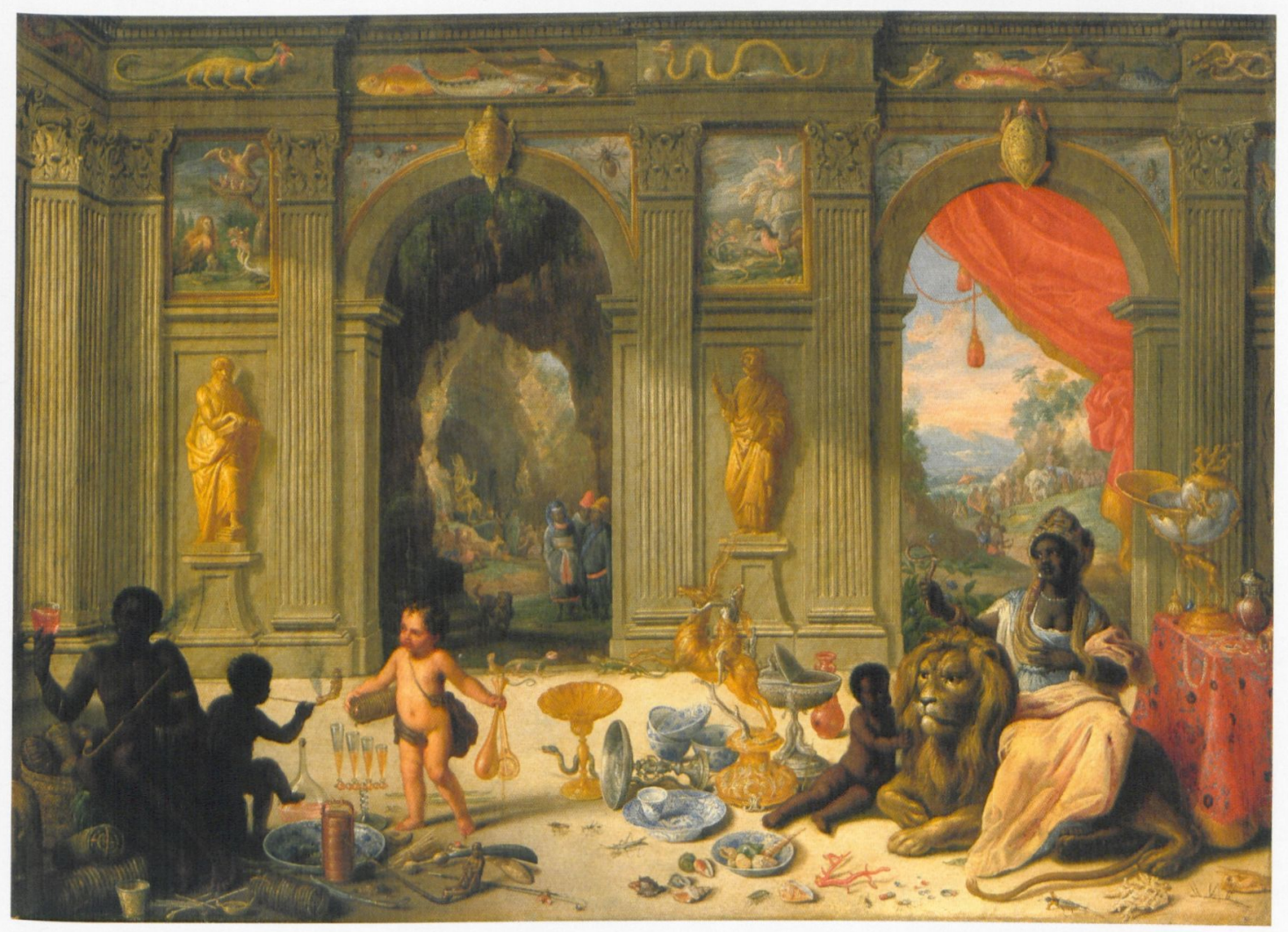

Abb. 20: Jan van Kessel, Afrika (Ausschnitt aus: Die vier Erdteile), 1664-1666, Öl auf Kupfer, $48,6 \times 67,8 \mathrm{~cm}$ (Mitteltafel), München, Bayerische Staatsgemäldesammlungen, Alte Pinakothek. 


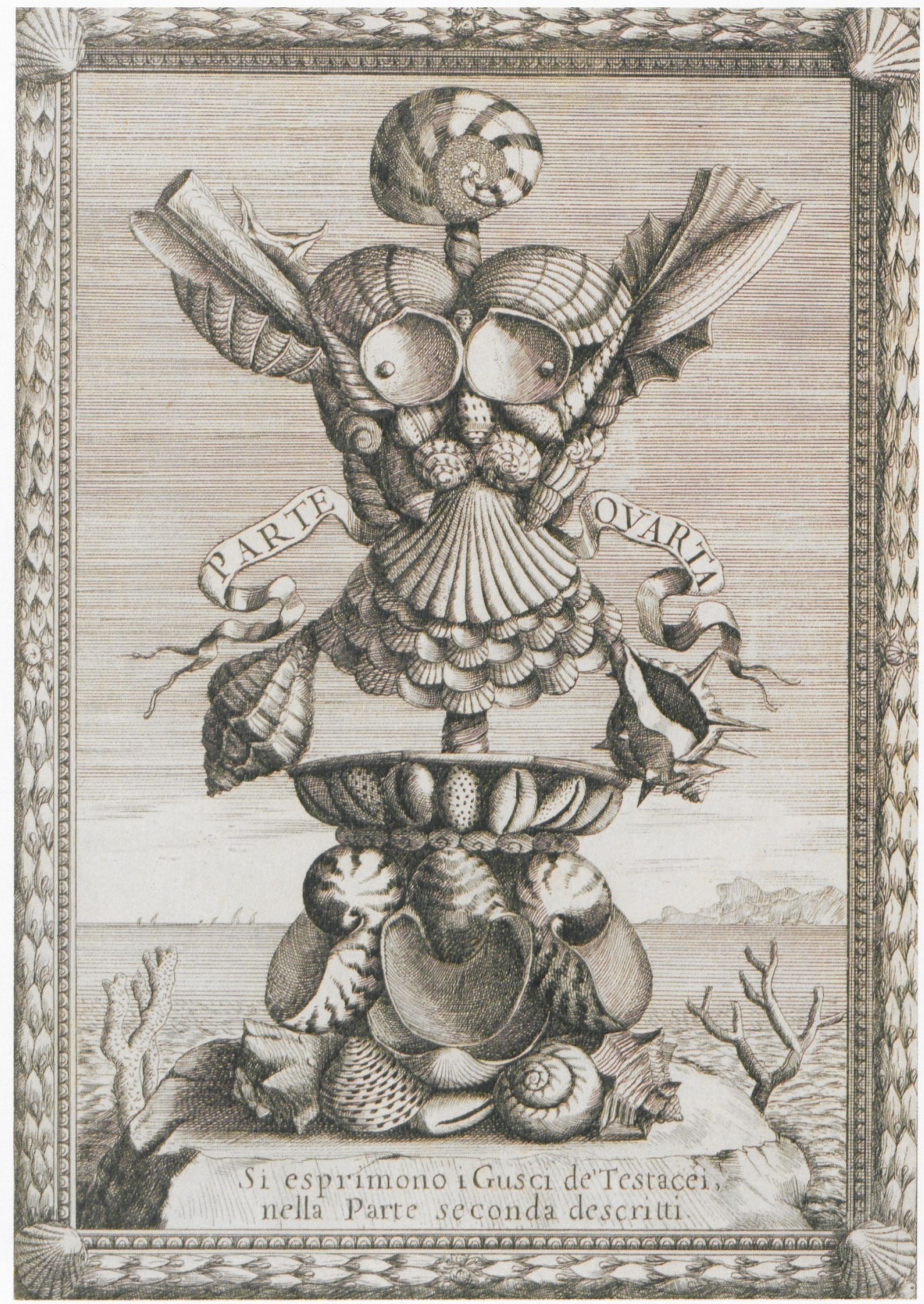

Abb. 21: Kupferstich, Titelblatt von Buonanni, Filippo (1681), Ricreatione dell'Occhio e della Mente nell' Osserveration' delle Chiocciole. Rom: Varese, Teil 4. 
es ihm primär um Vergnügen und Beobachtung, wie auch die zunehmend entstehenden reinen Muschelstillleben deutlich machen, auf die sich etwa Balthasar van der Ast (1590-1657) spezialisiert hat. Offenbar bricht sich hier die Vorstellung von der Legitimität der Neuzeit Bahn, in der die Neugierde ein Wert an sich ist und nicht mehr als Versuchung und Herausforderung des Göttlichen verstanden wird.

\section{Gleichberechtigung der Diskurse?}

Was lässt sich aus all dem Angeführten schliessen? Eine Bestätigung der Diskurstheorie, dass die verschiedensten Lektüren Sinn machen, keine die Deutungshoheit beanspruchen kann, eine Hierarchie der Sinnschichten nicht möglich, der Gegenstand per se polyvalent ist, er zudem in seiner Isolierung semiotisch unterdeterminiert ist, ohnedies keine klare Benennung zulässt? Jede Nachahmung ist Repräsentation, insofern ist sie durch einen vielfach bedingten Filter gegangen. Blosse Naturnachahmung als Selbstzweck gibt dem dargestellten Gegenstand noch keinen Kunstwert. Erst die Aufladung mit verweisendem Sinn, zumeist verbunden mit der Idealisierung der Form, stiftet dem Gegenstand Kunstcharakter. So wird man nur festhalten können: Die Grafik war Sammlerstück, legte Zeugnis von der Kunstfertigkeit ihres Entwerfers ab, der Gegenstand konnte aus den verschiedensten Blickwinkeln mit einem zeitgenössischen Interesse rechnen; er war exotisch genug, um kennerschaftliches Distinktionsstreben und -vergnügen auszulösen, die Konzentration auf ihn allein, allerdings in seiner atmosphärischen Erscheinung, erlöste ihn aus definitiven Verfügungszusammenhängen, seien sie religiös, naturwissenschaftlich, kulturgeschichtich oder ökonomisch. Was blieb, war das Bedürfnis, ihn zu betrachten, ja, wohl auch, ihn zu berühren, in die Hand zu nehmen, ihn hin- und herzuwenden, vielleicht auch ihn zu besitzen. Doch da es sich nur um eine Grafik handelt, bleibt es beim Betrachten, das nun allerdings unsere Bedürfnisse als Bedürfnisse bewusst macht, aber damit auch unser Sehen. Gerade das Dunkel, in das die Schnecke getaucht ist, suchen wir zu durchdringen, ohne zu wissen, was dahinter ist, doch darüber grübeln sollen wir schon - in diese oder jene der angeführten Richtungen.

Kunstgeschichte tendiert dazu, dem Dargestellten entweder definitiven Sinn beizumessen oder in das Gegenteil zu verfallen und es für gänzlich sinnoffen zu erklären. Damit würde es eine Tendenz zu reiner Sinnfreiheit aufweisen. Die Lösung pflegt dann zu sein, in ihm allein eine Demonstration seines Kunstcharakters zu sehen, als Selbstthematisierung der Kunst. Rembrandts Muschel könnte uns lehren, dass das eine wie das andere monokausal argumentiert. Kunstwerke haben ihren Kunstcharakter, aber auch ihren historischen Ort, sie bedingen einander. 
Abbildungsnachweis

Rijksmuseum, Amsterdam, Rijksprentenkabinet: Abb. 1, 2, 6, 17; () bpk - Bildagentur für Kunst, Kultur und Geschichte/ Kupferstichkabinett, Staatliche Museen zu Berlin/Volker H. Schneider: Abb. 3, 4; ( bpk - Bildagentur für Kunst, Kultur und Geschichte/Scala: Abb. 19; (c) bpk - Bildagentur für Kunst, Kultur und Geschichte/Bayerische Staatsgemäldesammlungen: Abb. 20; Herzog August Bibliothek Wolfenbüttel: Abb. 5; Fondation Custodia, Collection Frits Lugt, Paris: Abb. 7, 8, 15; Kunsthistorisches Museum, Wien: Abb. 9, 18; ETH Zürich, Sammlung Alte Drucke: Abb. 10; (c) The National Gallery, London: Abb. 11; Frans Hals Museum, Haarlem: Abb. 12; Rijksbureau voor Kunsthistorische Documentatie RKD: Abb.13, 14; Zentralbibliothek Zürich: Abb. 21.

\section{Bibliografie}

Barten, Sigrid (1985)

Die Muschel in der Kunst. Von der Renaissance bis zur

Gegenwart. Zürich: Kunstgewerbemuseum. (Ausstellungskatalog, Museum Bellerive Zürich)

\section{Bergström, Ingvar (1956)}

Dutch Still-Life Painting in the Seventeenth Century. New York: Yoseloff.

Beutelspacher, Albrecht \& Petri, Bernhard (1995)

Der Goldene Schnitt. Mannheim: BI Wiss.-Verlag.

Bevers, Holm, Schatborn, Peter \& Welzel, Barbara (Hrsg.) (1991) Rembrandt. Der Meister und seine Werkstatt. Zeichnungen und Radierungen. München: Schirmer-Mosel. (Ausstellungskatalog, Gemäldegalerie SMPK im Alten Museum, Berlin)
Busch, Werner (1994)

Der Berg als Gegenstand von Naturwissenschaft und Kunst. Zu Goethes geologischem Begriff. In Schulze, Sabine (Hrsg.), Goethe und die Kunst (S.485-518). Ostfildern: Hatje. (Ausstellungskatalog, Schirn Kunsthalle Frankfurt am Main/ Kunstsammlungen zu Weimar, Stiftung Weimarer Klassik)

Chong, Allan \& Klock, Wouter (Hrsg.) (1999) Still-Life Paintings from the Netherlands 1550-1720. Zwolle: Waanders. (Ausstellungskatalog, Rijksmuseum, Amsterdam/ The Cleveland Museum of Art)

Coomans, Henry E. \& Brus, René (1989)

Parels en Parelmoer. Scheveningen: Zeebiologisch Museum.

Coomans, Henry E. (1992)

Schelpenverzamelingen. In Bergvelt, Ellinor \& Kistemaker, Renée (Hrsg.), De wereld binnen handbereik. Nederlandse kunst und rariteitenverzamelingen, 1585-1735 (S.192-203). Zwolle: Waanders. (Ausstellungskatalog, Amsterdams Historisch Museum)

Flemming, Victoria von (1998)

Dissimulazione. Lorenzo Lippi, Salvator Rosa und die Krise der Repräsentation. In Göttler, Christine, Müller Hofstede, Ulrike, Patz, Kristina \& Zollikofer, Kaspar (Hrsg.), Diletto e Maraviglia. Ausdruck und Wirkung in der Kunst von der Renaissance bis zum Barock (S. 74-101). Emsdetten: Imorde.

Godfrey, Richard T. (Hrsg.) (1994)

Wenceslaus Hollar. A Bohemian Artist in England. New Haven: Yale University Press. (Ausstellungskatalog, Yale Center for British Art, New Haven)

Henkel, Arthur \& Schöne, Albrecht (Hrsg.) (1996)

Emblemata. Handbuch zur Sinnbildkunst des XVI. und XVII. Jahrhunderts. Stuttgart: Metzler. (Erstausgabe 1967) 
Hinterding, Erik, Luijten, Ger \& Royalton-Kisch, Martin (Hrsg.) (2000)

Rembrandt the Printmaker. Amsterdam: Waanders. (Ausstellungskatalog, The British Museum, London/Rijksmuseum, Amsterdam)

Jones, Barbara (1974)

Follies and Grottoes. London: Constable.

Langemeyer, Gerhard \& Peters, Hans-Albert (Hrsg.) (1979) Stilleben in Europa. Münster: Landschaftsverband WestfalenLippe. (Ausstellungskatalog, Westfälisches Landesmuseum für Kunst und Kulturgeschichte Münster/Staatliche Kunsthalle Baden-Baden)

Lindner, Gerd (1975)

Muscheln und Schnecken der Weltmeere. Aussehen, Vorkom. men, Systematik. München: BLV.

Mai, Ekkehard (1992)

Pictura in der "constcamer - Antwerpens Malerei im Spiegel von Bild und Theorie. In Mai, Ekkehard \& Vlieghe, Hans, Bruegel bis Rubens. Das goldene Jahrhundert der flämischen Malerei (S. 39-54). Köln: Wallraf-Richartz-Museum. (Ausstellungskatalog, Wallraf Richartz-Museum, Köln/Koninklijk Museum voor Schone Kunsten, Antwerpen/Kunsthistorisches Museum, Wien)

Mai, Ekkehard \& Vlieghe, Hans (1992)

Bruegel bis Rubens. Das goldene Jahrhundert der flämischen Malerei. Köln: Wallraf-Richartz-Museum. (Ausstellungskatalog, Wallraf Richartz-Museum, Köln/Koninklijk Museum voor Schone Kunsten, Antwerpen/Kunsthistorisches Museum, Wien)

Mauriès, Patrick (1994)

Shell Shock. Conchiological Curiosities. London: Thames and Hudson.
Meinhardt, Hans (2003)

The Algorithmic Beauty of Sea Shells. Berlin: Springer.

Mielke, Hans (Hrsg.) (1984)

Wenzel Hollar. Radierungen und Zeichnungen aus dem Berliner Kupferstichkabinett. Berlin: Staatliche Museen Preußischer Kulturbesitz. (Ausstellungskatalog, Kupferstichkabinett, Berlin)

Miller, Naomi (1982)

Heavenly Caves. Reflections on the Garden Grotto. Boston: George Allen and Unwin.

Müller Hofstede, Justus (1984)

»Non Saturatur Oculus Visu« - Zur »Allegorie des Gesichts« von Peter Paul Rubens und Jan Bruegel d. Ä. In Vekeman, Herman \& Müller Hofstede, Justus (Hrsg.), Wort und Bild in der niederländischen Kunst und Literatur des 16. und 17. Jahrhunderts (S. 243-89). Erftstadt: Lukassen Verlag.

Nieulandt, Adrian van (1970)

ljdelheid der ljdelheden. Hollandse Vanitas-Voorstellingen uit de zeventiende eeuw. Leiden: Stedelijk Museum De Lakenhal. (Ausstellungskatalog, Stedelijk Museum De Lakenhal, Leiden)

Pennington, Richard (1982)

A Descriptive Catalogue of the Etched Work of Wenceslaus Hollar. Cambridge: Cambridge University Press.

Sander, Jochen (1992)

Hugo van der Goes. Stilentwicklung und Chronologie (Berliner Schriften zur Kunst, Bd. 3). Mainz: Von Zabern.

Scheller, Robert W. (1969)

Rembrandt en de encyclopedische verzameling. Oud Holland, $84,2-3$, S. 81-147.

Schneider, Norbert (1989)

Stilleben. Realität und Symbolik der Dinge. Köln: Taschen. 
Seters, Wouter Hendrik van (1958)

Oud-Nederlandse parelmoerkunst. Het werk van leden der familie Belquin, parelmoergraveurs en schilders in de $17 \mathrm{e}$ eeuw. Nederlands Kunsthistorisch Jaarboek, 9, S. 173-238.

Strauss, Walter L. \& Meulen, Marjon van der (1979)

The Rembrandt Documents. New York: Abaris.

Vignau-Wilberg, Thea (2006)

Registrierender Blick und enzyklopädischer Geist: Der Miniaturist Joris Hoefnagel (1542-1600). In Lauterbach, Iris \& Stuffmann, Margret (Hrsg.), Aspekte deutscher Zeichenkunst (Veröffentlichungen des Zentralinstituts für Kunstgeschichte in München, Bd. 16) (S. 85-94). München: Zentralinstitut für Kunstgeschichte.

Visscher, Roemer (1614)

Sinnepoppen. Amsterdam: Willem lansz.

Volková, Jirina (Hrsg.) (1969)

Václav Hollar. Prag: Národní Galerie. (Ausstellungskatalog Národní Galerie, Prag)

Winner, Matthias (1957)

Die Quellen der Pictura-Allegorien in gemalten Bildergalerien des XVII. Jahrhunderts. Diss. Köln.
[1] Hinterding et al. (2000), S. 258-62, Kat. Nr. 62, hier die ausführlichste Analyse zu Rembrandts Grafik.

[2] Lindner (1975), S. 204, Taf. 47, Nr. 1.

[3] Besonders auffällig bei den Drei Kreuzen von 1653, wo in den ersten Zuständen der gute Schächer rechts von Christus am Kreuz im Schatten, der böse dagegen im Licht erscheint, entgegen der traditionellen ikonografischen Zuordnung von Sonne zum guten und Mond zum bösen Schächer, vgl. Hinterding et al. (2000), Kat. Nr. 73, 1. Zustand: Abb. S. 297, 3. Zustand: Abb. S. 298.

[4] Vgl. Pennington (1982), S. 337, Nr. 2187-2224, Godfrey (1994), S.19f. und 128f., Nr. 93; Abb. von Conus imperialis (Pennington (1982), Nr. 2195): Hinterding et al. (2000), S. 260, fig. a, oder von Cypraea tigris: Volková (1969), Kat. Nr. 243, oder von Trochus niloticus (heute: Tectus niloticus, zur Gattung der Kreiselschnecken gehörig): Mielke (1984), Abb. 120.

[5] Mielke (1984), Kat. Nr. 118, 119.

[6] Vgl. Barten (1985), Abb. 29, Kat. Nr. 21, Langemeyer et al. (1979), S. 174, Abb. 96 und 97, Vignau-Wilberg (2006), S. $85-94$.

[7] Siehe Anm. 4 .

[8] Vgl. Lindner (1975), S. 84 und 85.

[9] Hinterding et al. (2000), S. 261, Abb.d.

[10] Barten (1985), Abb. 30, Kat. Nr. 22.

[11] Von mathematischer Seite existieren erstaunliche Rechnungen von Muscheldekorationsformen und -proportionen: Meinhardt (2003), dort auch ausführlich zum Muster von Conus marmoreus, vgl. Index; die Teilung im Verhältnis des Goldenen Schnitts wird immer an den Nautilusschnecken demonstriert: Die innere Spirale verhält sich zur äusseren im Teilungsverhältnis des Goldenen Schnitts, vgl. Beutelspacher et al. (1995), S. 56.

[12] Vgl. Busch (1994), S. 485-97. 
[13] Vgl. Barten (1985), S. 110-13, Abb. 70, S. 112.

[14] Ebd., S. 202.

[15] Vgl. Bevers (1991), Kat. Nr. 29 der Radierungen, S. 248-50.

[16] Vgl. Pennington (1982), S. 337, vgl. auch Bergström (1956), S. $154-56$.

[17] Vgl. Lindner (1975), S. 41f. und 124, Taf. 7.

[18] Zu holländischen Muschelsammlungen vgl. Coomans (1992), S. 192-203; zur Perlmuttvorliebe der Holländer vgl. van Seters (1958), S. 173-238, Coomans et al. (1989); zu Goltzius' Allegorie vgl. Barten (1985), S. 121.

[19] Vgl. Barten (1985), S. 202-206, Kat. Nr. 116, Abb. S. 204, van Nieulandt (1970), Kat. Nr. 19; zu Harmen van Steenwyck vgl. Mauriès (1994), Abb.S. 31, van Nieulandt (1970), Kat. Nr. 29.

[20] Philibert van Borsselen, Strande oft Ghedichte van de schelpen, kinckhornen, ende andere wonderlicke Zee-schepselen. Amsterdam 1614, zit. nach Scheller (1969), S. 120.

[21] Visscher (1614), Teil 1, S. 5, Emblem 4; vgl. dazu auch Hollstein et al. (1991), Nr. 431, Chong et al. (1999), S.18, fig. 10; für das Emblem mit dem Motto "Tis misselijck waer een geck zijn gelt aen leijt ( (»Es ist sonderbar, wie ein Narr sein Geld spendet «) fertigte Claes Jansz. Visscher die Illustration an; vgl. dazu auch Bergström (1956), S. $156 \mathrm{f}$.

[22] Strauss et al. (1979), doc. 1656/12, Nr. 179.

[23] Vgl. Müller Hofstede (1984), S. 25 of.; vgl. auch S. 255-59, 266-68 und 279, Anm. 55 und 56. Sowohl in Rubens' und Jan Brueghels d. Ä. Allegorie des Gesichts wie im zugehörigen Bild Gesicht und Geruch werden die Muscheln mit Bezug auf Roemer Visscher in malo gedeutet. Vgl. Mai (1992), S. 48-50.

[24] Vgl. Mai et al. (1992), S. 371f., Kat. Nr. 52.1.

[25] Vgl. Winner (1957).

[26] Vgl. van Borsselen (wie Anm. 20); dazu Scheller (1969), S. 120 .
[27] So Ekkehard Mai, vgl. Mai et al. (1992), S. 372.

[28] Zu den Begriffen vgl. etwa Flemming (1998), S. 74-101.

[29] Vgl. Bevers (1991), S. 242-45, Kat. Nr. 27 der Radierungen, Hinterding et al. (2000), S. 253-58, Kat. Nr. 61.

[30] Vgl. zum Wiener Diptychon Sander (1992), S. 44-90. Die folgende Beobachtung scheint der Forschung nicht aufgefallen zu sein.

[31] Vgl. Lindner (1975), S. 30 f., 35 und Taf. 1, S. 112, Nr. 1, 1 a.

[32] Abb. in Mauriès (1994), S. 27.

[33] Mai et al. (1992), Kat. 35.2, Abb. S. 325, Schneider (1989), Kap. 12, S. 157-70, Detailabb. von »Amerika« S. 166, von "Afrika« S. 167 .

[34] Henkel et al. (1996), Sp. 361-63.

[35] Vgl. Miller (1974), Mauriès (1994), S. 50-73.

[36] Mauriès (1994), Abb. S. 29. 